\title{
ADAMTS-13 in the Diagnosis and Management of Thrombotic Microangiopathies
}

\author{
Galit Sarig, Ph.D.* \\ Hematology Laboratory, Rambam Health Care Campus; and Bruce Rappaport Faculty of Medicine, \\ Technion, Israel Institute of Technology; Haifa, Israel
}

\begin{abstract}
Thrombotic microangiopathies (TMAs) comprise a group of distinct disorders characterized by microangiopathic hemolytic anemia, thrombocytopenia, and microvascular thrombosis. For many years distinction between these TMAs, especially between thrombotic thrombocytopenic purpura (TTP) and hemolytic uremic syndrome (HUS), remained purely clinical and hard to make. Recent discoveries shed light on different pathogenesis of TTP and HUS. Ultra-large von Willebrand factor (UL-VWF) platelet thrombi, resulting from the deficiency of cleavage protease which is now known as ADAMTS-13 (a disintegrin and metalloproteinase with a thrombospondin type 1 motif, member 13), were found to cause TTP pathology, while Shiga toxins or abnormalities in regulation of the complement system cause microangiopathy and thrombosis in HUS. TMAs may appear in various conditions such as pregnancy, inflammation, malignancy, or exposure to drugs. These conditions might cause acquired TTP, HUS, or other TMAs, or might be a trigger in individuals with genetic predisposition to ADAMTS-13 or complement factor $\mathrm{H}$ deficiency. Differentiation between these TMAs is highly important for urgent initiation of appropriate
\end{abstract}

\begin{abstract}
Abbreviations: ADAMTS-13, a disintegrin and metalloproteinase with a thrombospondin type 1 motif, member 13; aHUS, atypical hemolytic uremic syndrome; CV, coefficient of variation; DIC, disseminated intravascular coagulopathy; ECAT, External Quality Control for Assays and Tests with a focus on Thrombosis and Haemostasis; ELISA, enzyme-linked immunosorbent assay; FRET, fluorescence resonance energy transfer; GP, glycoprotein; HELLP syndrome, hemolysis, elevated liver enzymes, and low platelets syndrome; HUS, hemolytic uremic syndrome; LDH, lactate dehydrogenase; PEX, plasma exchange; Stx, Shiga toxin; TMAs, thrombotic microangiopathies; UL-VWF, ultra-large von Willebrand factor; VWF, von Willebrand factor.
\end{abstract}

Citation: Sarig G. ADAMTS-13 in the Diagnosis and Management of Thrombotic Microangiopathies. Rambam Maimonides Med J 2014;5 (4):eoo26. doi:10.5041/RMMJ.10160

Copyright: (C) 2014 Sarig. This is an open-access article. All its content, except where otherwise noted, is distributed under the terms of the Creative Commons Attribution License (http://creativecommons.org/licenses/by/3.0), which permits unrestricted use, distribution, and reproduction in any medium, provided the original work is properly cited.

Conflict of interest: No potential conflict of interest relevant to this article was reported.

* E-mail: g_sarig@rambam.health.gov.il 
therapy. Measurement of ADAMTS-13 activity and anti-ADAMTS-13 antibody levels may advance this differentiation resulting in accurate diagnosis. Additionally, assessment of ADAMTS-13 levels can be a tool for monitoring treatment efficacy and relapse risk, allowing consideration of therapy addition or change. In the past few years, great improvements in ADAMTS-13 assays have been made, and tests with increased sensitivity, specificity, reproducibility, and shorter turnaround time are now available. These new assays enable ADAMTS-13 measurement in routine clinical diagnostic laboratories, which may ultimately result in improvement of TMA management.

KEY WORDS: ADAMTS-13, aHUS, HUS, thrombotic microangiopathies, TTP, UL-VWF, Von

Willebrand factor

\section{INTRODUCTION}

Thrombotic microangiopathies (TMAs) comprise a group of distinct disorders characterized by microangiopathic hemolytic anemia, thrombocytopenia, and microvascular thrombosis, regardless of cause or specific tissue involvement. ${ }^{1}$ The pathogenesis is associated with thrombus formation in the microvasculature of various organs, which leads to a consumptive thrombocytopenia and creates an abnormally high level of shear stress in the small vessels. ${ }^{2,3}$ The shear stress eventually leads to mechanical destruction of erythrocytes and the presence of fragmented erythrocytes (schistocytes) in the peripheral blood.

Thrombotic microangiopathies may result from four types of lesions: ultra-large von Willebrand factor (UL-VWF)-platelet thrombi, as in thrombotic thrombocytopenic purpura (TTP); fibrin-platelet thrombi, as exemplified by disseminated intravascular coagulopathy (DIC) and catastrophic antiphospholipid syndrome; inflammatory or proliferative microangiopathy accompanied by variable fibrin thrombi, as in hemolytic uremic syndrome (HUS); and intravascular clusters of cancer cells. 4

For many years, the distinction between these TMAs remained purely clinical, and this led to the belief that these disorders were different manifestations of the same pathological process. However, recent advances have demonstrated that UL-VWFplatelet thrombi in TTP result from the deficiency of a VWF cleavage protease which is now known as ADAMTS-13 (a disintegrin and metalloproteinase with a thrombospondin type 1 motif, member 13), whereas microangiopathy and thrombosis as in HUS result mainly from exposure to Shiga toxins or abnormalities in regulation of the complement system. 4
To understand differences in pathophysiology of TMAs, this review describes the history of TMA and ADAMTS-13 discovery, various TMAs, and the way to differentiate between them. In addition, the article discusses the importance of rapid ADAMTS13 evaluation to ensure an accurate diagnosis and urgent initiation of the appropriate therapy. The role of ADAMTS-13 status in risk assessment and monitoring response to treatment is also addressed.

\section{THE HISTORY OF TMA PATHOGENESIS DISCOVERY -FROM BEDSIDE TO BENCH}

The history of the TMA discovery (between 1924 and 1960 ) is associated with very talented clinicians who had the ability and the vision to recognize the pathophysiology of the diseases, although they lacked the technology to demonstrate and prove it. Not until the 1980 os was evidence for the proposed mechanisms discovered.

In 1924, Eli Moschcowitz was the first to report on a 16-year-old girl with a sudden onset of fever and hemolytic anemia, followed rapidly by paralysis, coma, and death. Moschcowitz suspected that microvascular platelet-rich thrombi which were found in the microcirculation were the cause for this disease. 5 This was probably the first description of TTP.

In 1955, Gasser et al. described five childhood cases of HUS, which were clinically defined by thrombocytopenia, non-immune microangiopathic hemolytic anemia, and acute kidney failure followed by death from renal cortical necrosis. ${ }^{6}$

In 1960, Schulman et al. described the case of an 8-year-old girl who exhibited relapsing episodes of thrombocytopenia. This patient responded well to plasma infusion, and the authors suggested that the 
disorder was due to the deficiency of a plateletstimulating factor. Upshaw later reported comparable findings in a 29-year-old woman whose first episode occurred at the age of 6 months. The author suggested as the underlying pathogenic mechanism the deficiency of a plasma factor that promotes platelet and red blood cell destruction. ${ }^{7}$ It is now clear that the disorder described by Schulman and Upshaw, which now bears their name-the Upshaw-Schulman syndrome-represents a congenital form of TTP.

In 1982, Moake et al. found ultra-large molecular forms of von Willebrand Factor (UL-VWF) in patients with TTP and proposed that this played a pathogenic role in the formation of microvascular platelet-rich thrombi in patients with acute TTP. ${ }^{8}$

In 1985, Karmali et al. discovered the link between the HUS and enteric infections with Escherichia coli that produce Shiga toxin (Stx). ${ }^{9}$

In 1996, simultaneously, Furlan et al. in Switzerland and Tsai in New York reported independently the isolation and identification of a VWFcleaving protease from human plasma. ${ }^{10,11}$

In 2001, several groups (Fujikawa et al., Gerritsen et al., and Levy et al.) identified the VWFcleaving protease as ADAMTS-13. ${ }^{12-14}$

The majority of patients with TTP show severe deficiency in the VWF-cleaving activity of ADAMTS13, either caused by missense or frame-shift mutations ${ }^{14-16}$ (Figure $1 \mathrm{~B}$ ) or due to ADAMTS-13 neutralizing autoantibodies. ${ }^{17,18}$

An ADAMTS-13 deficiency and defective complement regulation have been identified as the two major causes of TMA. It is now possible to classify TMA pathogenetically rather than just clinically.

Recently, it was shown that the coagulation cascade, platelet activation, ADAMTS-13 activity, and UL-VWF multimers are associated with the complement pathway regulation. ${ }^{19}$ The role of these interrelationships in TMAs should be further studied to improve TMA management.

\section{VON WILLEBRAND FACTOR AND ULTRA- LARGE MULTIMERS OF VWF}

Von Willebrand factor (VWF) is a large complex of multimeric molecules, range $0.8-20 \times 10^{6} \mathrm{kDa}$, encoded on chromosome 12. Synthesis of VWF is by endothelial cells and megakaryocytes and stored in Weibel-Palade bodies in endothelial cells and in platelet $\alpha$ granules. Secretion is induced by vascular injury and stimuli by thrombin, histamine, vasopressin, inflammatory cytokines, and Stx.

Von Willebrand factor is released from endothelial cells as ultra-large multimers of VWF (UL-VWF) which can bind the glycoprotein (GP) Iba components of platelet GPIb-IX-V receptor and induce platelet adhesion and aggregation by shear stress in the arterioles and capillaries (Figure 1B). ${ }^{20,21}$ These microvascular thrombi of platelets with UL-VWF result in platelet consumption and hemolysis and cause thrombotic microangiopathy of several TMAs. ${ }^{22}$

In normal subjects, the UL-VWF molecules are cleaved by a specific VWF-cleaving metalloprotease present in the plasma, ADAMTS-13 (Figure 1A). ${ }^{10-14}$ The enzyme degrades UL-VWF multimers by cleaving $1605 \mathrm{Tyr}-1606 \mathrm{Met}$ peptide bonds in susceptible A2 domains of VWF monomeric subunits. ${ }^{1}$

\section{THE STRUCTURE OF ADAMTS-13}

ADAMTS-13 is a disintegrin and metalloprotease with eight thrombospondin-1-like domains (Figure 2) composed of an amino-terminal reprolysin-type metalloprotease domain followed by a disintegrin domain, a thrombospondin-1-like domain, a cysteine-rich domain containing an arginineglycine-aspartate sequence and an adjacent spacer portion, seven additional thrombospondin-1-like domains, and two similar CUB domains at the carboxyl-terminal end of the molecule. The CUB domains, found only in ADAMTS-13 among the ADAMTS enzyme family members, contain peptide sequences present in complement subcomponents C1r/C1s, embryonic sea urchin protein, and bone morphogenic protein-1.23

The spacer and CUB-1 domains are involved in binding ADAMTS-13 to the UL-VWF secreted by endothelial cells. ${ }^{24}$ The ADAMTS-13 enzymes attach through spacer/CUB domains to accessible A3 domains in the monomeric subunits of the VWF strings, ${ }^{25}$ and then cleave a $1605 \mathrm{Tyr}-1606 \mathrm{Met}$ peptide bond in an adjacent VWF A2 domain.

ADAMTS-13 is a $\mathrm{Zn}^{2+}$ and $\mathrm{Ca}^{2+}$-requiring 190,000-dalton glycosylated protein that is encoded on chromosome 9q34 and produced predominantly in the liver. The activity of ADAMTS-13 is inhibited in vitro by ethylenediaminetetra-acetic acid (EDTA), and, therefore, functional assays of the enzyme are usually performed using plasma anticoagulated with 

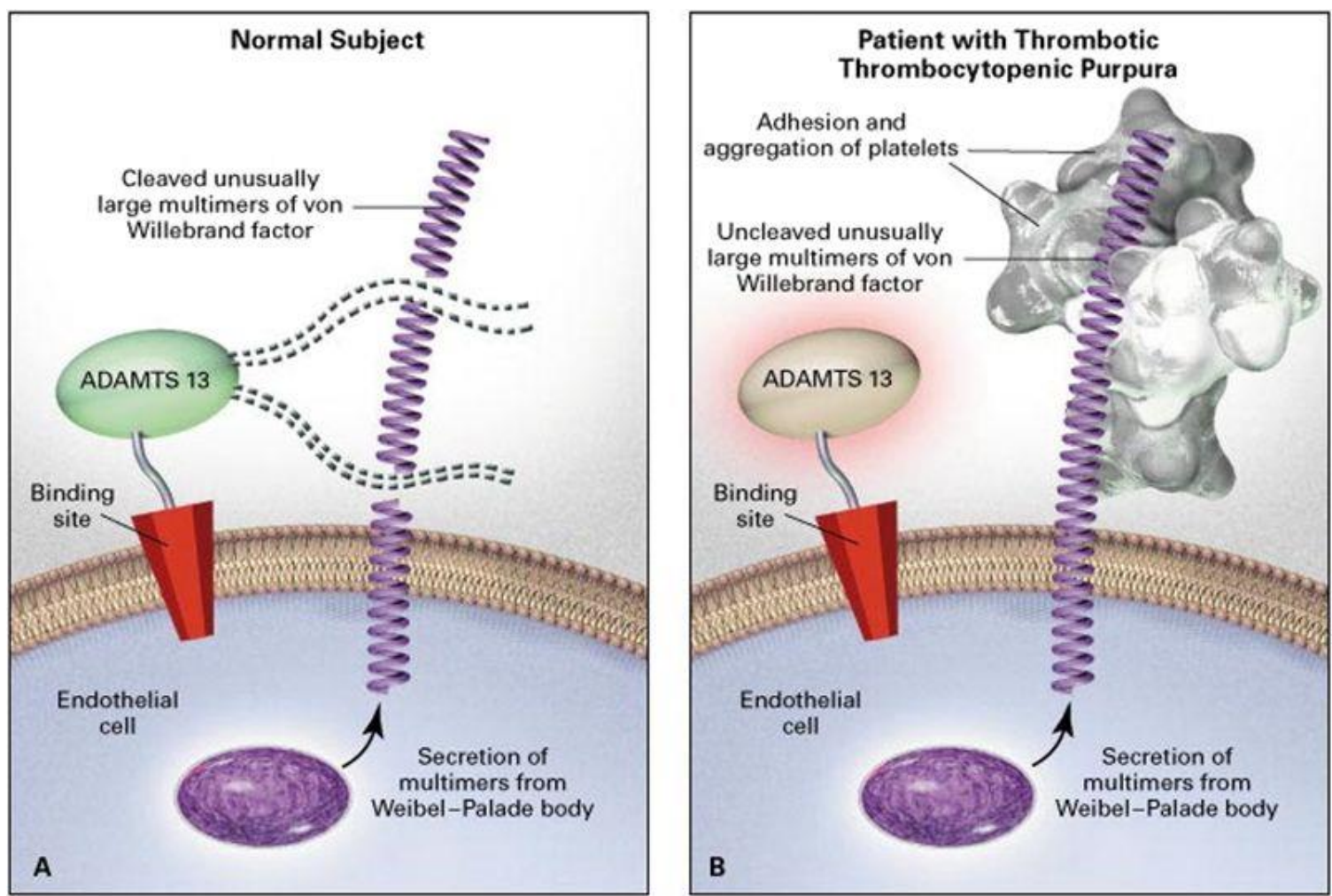

Figure 1. Proposed Relation among the Absence of ADAMTS-13 Activity In Vivo, Excessive Adhesion and Aggregation of Platelets, and Thrombotic Thrombocytopenic Purpura.

In Panel A, in normal subjects, ADAMTS-13 (von Willebrand factor-cleaving metalloprotease) molecules attach to binding sites on endothelial cell surfaces and cleave unusually large multimers of von Willebrand factor as they are secreted by stimulated endothelial cells. The smaller von Willebrand factor forms that circulate after cleavage do not induce the adhesion and aggregation of platelets during normal blood flow. The ADAMTS-13 may use one of its thrombospondin-1-like domains or its arginine-glycine-aspartate (RGD) sequence to attach to the surface of endothelial cells.

In Panel B, absent or severely reduced activity of ADAMTS-13 in patients with thrombotic thrombocytopenic purpura prevents timely cleavage of unusually large multimers of von Willebrand factor as they are secreted by endothelial cells. The uncleaved multimers induce the adhesion and aggregation of platelets in flowing blood. A congenital deficiency of ADAMTS-13 activity or an acquired defect of ADAMTS-13 (such as that caused by autoantibodies or by a change in the production or survival of the protein) can lead to thrombotic thrombocytopenic purpura. Interference with the attachment of ADAMTS-13 to endothelial cells in vivo (for example, as a result of ADAMTS-13-receptor blockade by other types of autoantibodies) may also cause thrombotic thrombocytopenic purpura in patients with normal ADAMTS-13 activity in plasma.

From: Moake JL. Thrombotic microangiopathies. N Engl J Med 2002;347:587-600. Copyright @ Massachusetts Medical Society. Reprinted with permission from Massachusetts Medical Society.

citrate. ${ }^{10,11}$ Anti-ADAMTS-13 antibodies preferentially bind to the cysteine-rich and spacer regions of the ADAMTS-13 molecule.

\section{THROMBOTIC MICROANGIOPATHY}

Thrombotic microangiopathy (TMA) refers to a group of pathological disorders that are characterized by hemolytic anemia, thrombocytopenia, and widespread microvasculopathy, with or without thrombi. 4

Clinical manifestations of TMA reflect ischemic injury of the affected organs. In some patients neurological deficits predominate; in others, renal failure is severe. This clustering provided a convenient basis for defining thrombotic thrombocytopenic purpura (TTP) and the hemolytic uremic syndrome 


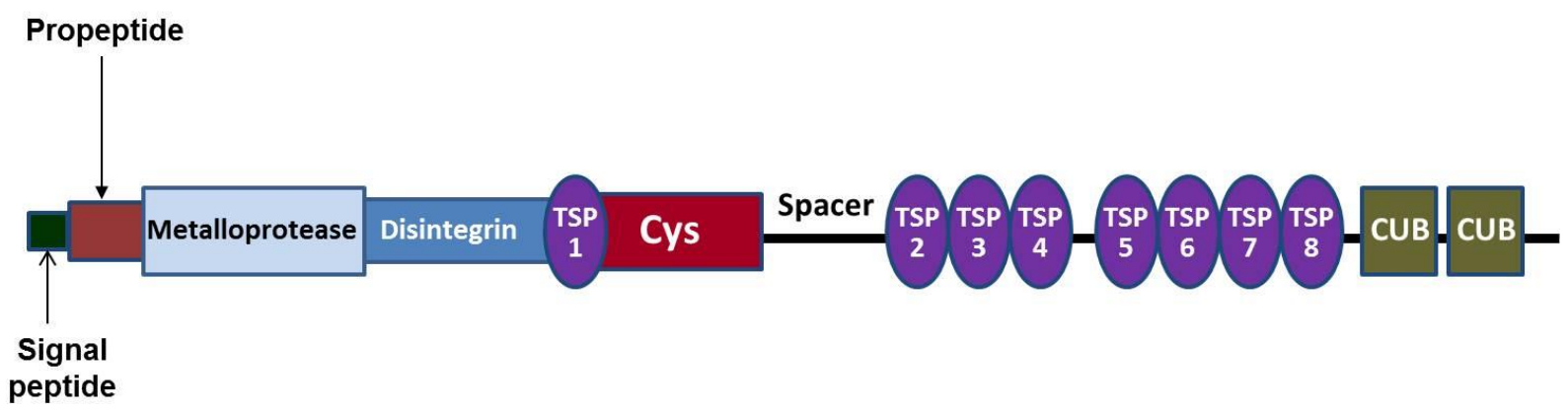

Figure 2. ADAMTS-13 Domain Structure.

ADAMTS-13 N-terminal composed of a signal peptide and propeptide, which are cleaved during the protein processing. The catalytic domain consists of the metalloprotease and disintegrin domains. A first thrombospondin-1 (TSP 1) is followed by a cysteine-rich domain (Cys), a spacer, and sevenadditional TSP 1 repeats (TSP 2-8). The C terminus contains two complement components $\mathrm{C} 1 \mathrm{r} / \mathrm{C} 1 \mathrm{~s}$, embryonic sea urchin protein, and bone morphogenic protein-1(CUB) domains.

(HUS). However, this classification has been misleading, since some patients have both neurological deficits and renal failure, and others may have predominant neurological deficits or renal failure on different occasions.

Thrombotic microangiopathy may appear in a variety of conditions such as pregnancy, inflammation, malignancy, or exposure to such drugs as thienopyridines or calcineurin inhibitors. These conditions might be the cause of acquired TTP, HUS, or HELLP syndrome (hemolysis, elevated liver enzymes, low platelets), or the trigger in individuals with a genetic predisposition to ADAMTS-13 or complement factor $\mathrm{H}$ deficiency.

While patients with congenital TTP and acquired immune TTP attributed to low ADAMTS-13 activity demonstrate a good response to plasma infusion or plasma exchange (PEX), other clinical forms of TMA occur in the absence of severe ADAMTS-13 deficiency, and this may be the reason why patients with the other clinical forms of TTP do not respond to plasma therapy. ${ }^{26}$

The diagnosis of TMA can be very difficult, as there is a clinical overlap between various TMAs. Since in untreated cases mortality may approach $90 \%$, the availability of ADAMTS-13 activity and anti-ADAMTS-13 antibody assays is crucial for the differentiation between the TMAs, accurate diagnosis, and urgent initiation of the appropriate treatment.

\section{Thrombotic Thrombocytopenic Purpura}

Thrombotic thrombocytopenic purpura (TTP) is rare, with a reported incidence of 4-6 cases per million per year and with a female-to-male ratio of $3: 2 .{ }^{27,28}$ In spite of major progress in early detection and modern therapies, early death still occurs: approximately half of the deaths in the regional UK TTP registry occurred within 24 hours of presentation. ${ }^{27}$

Thrombotic thrombocytopenic purpura is characterized by microvascular platelet clumping, resulting in microangiopathic hemolytic anemia, fragmented erythrocytes (schistocytes), consumptive thrombocytopenia, renal dysfunction, and neurological symptoms. However, TTP can present without the full pentad; up to $35 \%$ of patients do not have neurological signs at presentation, and renal abnormalities and fever are not prominent features. The revised diagnostic criteria state that TTP must be considered in the presence of thrombocytopenia and microangiopathic hemolytic anemia alone.29 The diagnosis of TTP remains based on clinical history, examination of the patient, and the blood film. Assays for ADAMTS-13 help to confirm the diagnosis, differentiate TTP from other TMA forms, and monitor the efficacy of treatment. They are also helpful in consideration of the need for additional or alternative therapy.

In general, congenital TTP is defined by ADAMTS-13 deficiency, while acquired TTP is 
defined by the presence of ADAMTS-13 neutralizing autoantibodies. During an acute episode, before starting therapy, ADAMTS-13 activity level of $<5 \%$ supports the TTP diagnosis.

\section{Congenital TTP: Upshaw-Schulman Syndrome}

This very rare condition with a prevalence of about $0.05-0.4$ per 100,000 is transmitted by autosomal recessive inheritance. $3^{30,31}$ Neonates with severe phenotype typically have major neonatal jaundice. Blood film examination may show schistocytes together with red cell anisocytosis. ${ }^{2}$ More frequently, the diagnosis is made later in infancy or childhood, 33 typically with thrombocytopenia, microangiopathic hemolytic anemia, jaundice, and elevated lactate dehydrogenase (LDH), although some children may only have an isolated thrombocytopenia. Neurological symptoms, such as hemiparesis, hemiplegia, or seizures, occur in $35 \%$ of cases. 34

Patients presenting during adulthood tend to have milder clinical courses. ${ }^{3}$ However, the clinical course in individual patients can be highly variable. ${ }^{30}$ The presence of an affected sibling or a therapeutic response to a plasma-containing blood product may propose the diagnosis. 35

The diagnosis of congenital TTP is dependent on detecting ADAMTS- 13 activity of $<5 \%$ during an acute episode, in the absence of antibodies to ADAMTS-13. Over the last few years, molecular analysis has been used to confirm the diagnosis, and either a homozygous or compound heterozygote defect in ADAMTS-13 could be found. Testing of siblings and other first-degree relatives at risk should be considered. ${ }^{36}$

Mutations affecting the highly conserved Nterminal domains of ADAMTS-13 are associated with lower residual ADAMTS-13 activity and a more severe clinical phenotype in an allelic dosedependent manner. 37,31 Mutations located in the Cterminal part of ADAMTS-13 are associated with a less severe clinical expression.

A mutation located in the TSP1-7 domain, p.Arg1060Trp, is specifically associated with an adult onset of congenital TTP and found with a very high prevalence in women in whom TTP events are associated with pregnancy. ${ }^{3}$

Patients with congenital TTP have persistently low levels of ADAMTS-13, but they can be asymptomatic until a further precipitating event results in an acute TTP episode. Events include febrile episodes, infections, vaccinations, excess alcohol intake, and in females mostly pregnancy. ${ }^{29-41}$

Rarely, patients with a "late-onset phenotype" may not develop symptoms until their 50 os or 6os with isolated cerebral events or renal disease. ${ }^{42}$ Asymptomatic male cases are usually detected because they have affected siblings.

\section{Acquired Thrombotic Thrombocytopenic Purpura}

Acquired TTP is a rare, autoimmune disease characterized by antibodies, usually IgG, directed against ADAMTS-13, with an annual incidence of $0.2-1$ per $100,000.43$ In its most common, characteristic form, TTP begins abruptly and virulently, occasionally after a febrile, viral-like prodrome; a minor infection or pregnancy may be the trigger.44,45 Thrombocytopenia and fragmentation hemolysis are severe, and central neurologic signs exist at presentation or supervene quickly, out of proportion to renal signs. Dialysis-requiring renal failure is rare. Without immediate recognition and intervention, death, often precipitated by seizures and arrhythmias, may come rapidly and suddenly. Before the advent of modern therapy, mortality was about $90 \%$. One-third of TTP survivors experience relapses over the course of years, especially soon after initial presentation. ${ }^{46,47}$ Some have persistent cognitive and central neurologic impairments, other chronic health problems, or die prematurely even when TTP is inactive. $4^{8-50}$ About $5 \%-10 \%$ of patients later in their course manifest systemic lupus erythematosus. $5^{\circ}$

\section{Hemolytic Uremic Syndrome}

Hemolytic uremic syndrome (HUS) is a TMA defined by thrombocytopenia, microangiopathic hemolytic anemia, and acute renal failure with elevated serum creatinine levels, low glomerular filtration rates, microscopic hematuria, and subnephrotic proteinuria. ${ }^{51}$ The most frequent form is associated with infections by Shiga-like toxin-producing bacteria (Shiga-HUS). Atypical form of HUS (aHUS) is associated with defects in the immunological complement pathway.

Shiga toxins (Stx)-1 and Stx-2, produced by enterohemorrhagic $E$. coli, stimulate rapid and profuse secretion of UL-VWF from endothelial cells, in particular glomerular microvascular endothelial cells. ${ }^{53}$ Platelets immediately adhere to the secreted 
UL-VWF, and the rate of platelet-VWF string cleavage by ADAMTS- 13 is delayed in the presence of Stx-1 or Stx-2. This may explain the glomerular microvascular occlusion and acute renal failure. $5^{2}$

Hemolytic uremic syndrome usually occurs as a single episode, except for rare individuals who have a familial, recurrent type of the disease.,23 In these patients, often children, with aHUS, the level of the plasma complement control protein, factor $\mathrm{H}$, is abnormally low most of the time. The result is overactivation of complement component $3\left(\mathrm{C}_{3}\right)$, which causes permanent glomerular endothelial activation and obstruction of the glomerular microvasculature by platelet-fibrin thrombi. ${ }^{23}$

\section{Shiga Toxin-induced HUS}

About $10 \%-20 \%$ of symptomatic infections lead to HUS. ${ }^{23,53}$ Shiga toxin-induced HUS (Shiga-HUS) is the commonest TMA, most prevalent in children under the age of 5 years, with an annual incidence of 6 per 100,000.54 Severe thrombocytopenia, fragmentation hemolysis, renal failure, and hypertension are characteristic.

The diagnosis of Shiga-HUS depends on the detection of $E$. coli $\mathrm{O} 157: \mathrm{H} 7$ and other Stxproducing bacteria and their products in stool cultures. ${ }^{1}$

In extreme cases, the brain and other organs may be involved. The condition is a medical emergency with a short-term mortality of about $5 \%-10 \%$ without urgent therapy. Renal function recovers in $70 \%$ to over $90 \%$ of cases. 35

Approximately $5 \%$ of HUS cases in children are not associated with Stx-producing bacteria and result from infection by neuraminidase-producing Streptococcus pneumoniae. ${ }^{51}$

\section{Atypical HUS}

Cases of atypical HUS (aHUS) are rare, one-tenth as frequent as Shiga-HUS. 54 The first presentations are most of the time in children, including neonates, but may not occur until later in life. About $20 \%$ are familial phenomena. Infections and pregnancy may trigger an acute episode. 54 The end-organ presentation is predominantly renal, but cardiac, neurologic, and more rarely large artery obstruction may occur. The prognosis before recent treatment advances was poorer than for Shiga-HUS, with over half of cases progressing to end-stage renal failure and onequarter of patients dying of the disease. 35
Atypical HUS is the result of excessive alternative complement pathway activation.

Prominent causes of aHUS are a heterozygous mutation of the complement factor $\mathrm{H}$ gene, or homozygous deletion in genes for the factor $\mathrm{H}$ related proteins or autoantibody-mediated inhibition of factor $\mathrm{H}$ deficiency.

Other mutations associated with aHUS include heterozygous loss-of-function mutations of complement factor I, membrane co-factor protein or $\mathrm{CD} 46$ or thrombomodulin, heterozygous gain-of-function mutations in $\mathrm{C}_{3}$ or complement factor $\mathrm{B}$.

Excessive alternative complement pathway activity in aHUS results in severe damage of renal endothelial cells, leading to renal failure. ${ }^{19}$

Lately, treatment with eculizumab has been approved for aHUS. Eculizumab is a humanized monoclonal antibody to terminal complement protein $\mathrm{C}_{5}$ that prevents activation of the terminal complement pathway by binding $\mathrm{C}_{5}$ and inhibiting generation of pro-inflammatory $\mathrm{C}_{5} \mathrm{a}$ and the lytic C5b-9 membrane attack complex. Before administering eculizumab therapy for an acute episode of aHUS, there is a need to rule out TTP which is proved by normal levels of ADAMTS-13 activity (>30\%), without the presence of anti-ADAMTS-13 antibodies.

\section{Pregnancy-associated TMA}

Pre-eclampsia and HELLP syndrome are serious TMA complications in pregnancy. In these events, the hypoxic placenta releases receptors for angiogenic factors, like soluble VEGF receptor-1. These circulating soluble angiogenic receptors contribute to the progressive renal dysfunction and hepatic necrosis in pregnancy TMAs. 55

Nevertheless, pregnancy is a hypercoagulation state with very high levels of VWF and UL-VWF released from endothelial cells and the placenta, and can trigger TTP (acquired or congenital), aHUS, or other TMAs.

Pregnancy is the initiating event for approximately $5 \%-25 \%$ of TTP cases, which are late-onset adult congenital TTP or acute idiopathic TTP. ${ }^{27,36}$

Thrombosis occurs in the placenta in untreated TTP pregnancies, resulting in fetal growth restriction, intrauterine fetal death, and pre-eclampsia. There is a continued risk of relapse during subsequent pregnancies. However, there are some 
reports demonstrating that women with normal prepregnancy levels of ADAMTS-13 have a lower risk of relapse.56,57 Differentiating TTP from more common pregnancy-related TMAs is difficult. In these suspected cases, measurement of ADAMTS-13 activity and anti-ADAMTS-13 antibodies can advance the differentiation. Low ADAMTS-13 activity and the presence of anti-ADAMTS-13 antibodies can distinguish congenital and acquired TTP, respectively, from other pregnancy-associated TMAs. Although in pre-eclampsia and HELLP syndrome ADAMTS-13 activity is reduced (median 31\%, range $12 \%-43 \%$ ), in an acute TTP episode the ADAMTS-13 activity levels are below $10 \%$. In addition, presence of anti-ADAMTS-13 antibodies supports the diagnosis of acquired TTP. ${ }^{36}$

\section{TMA Due to Drugs}

Thienopyridines (ticlopidine and clopidogrel) are the most frequent TMA-causing drugs reported to the United States Food and Drug Administration. Ticlopidine, now infrequently prescribed, produced the highest incidence, 1 in 1,600 to 5,000 patients. About 90\% of cases occurred between 2 and 12 weeks of therapy. Clopidogrel causes TMA less frequently, about 1 in 80,000 patients.58,59 Other drug causes are the calcineurin inhibitors (cyclosporine and tacrolimus), the mTOR inhibitors (sirolimus and everolimus), anti-neoplastic agents (mitomycin and gemcitabine, both in a cumulative, dose-dependent manner), and quinine. 35

Drugs appear to be responsible for $<15 \%$ of all TTP cases. Ticlopidine therapy increases the risk of developing ADAMTS-13 inhibitors 200- to 300fold. ${ }^{60,61}$

Some chemotherapy agents, such as gemcitabine, bleomycin, and mitomycin-C, can cause HUS but not TTP. ${ }^{36}$

\section{Transplant and Malignancy-associated TMA}

Transplant-associated TMA is a microangiopathy hemolytic anemia with thrombocytopenia that occurs after bone marrow transplantation. It may reflect endothelial toxicity associated with chemotherapy, infections, immunosuppressives, and graftversus-host disease (GVHD).35,62

Thrombotic microangiopathy occurs in association with a variety of malignancies, especially adeno- carcinomas. ${ }^{63}$ Presentation may be either at an early stage of cancer or associated with disseminated disease.

The ADAMTS- 13 activity is not significantly reduced in transplant and malignancy-associated TMA, 64 and this might explain the inefficiency of PEX therapy in these patients. Some suggest that due to endothelial damage associated with malignancy, chemotherapy, and bone marrow transplantation, there is an extreme release of UL-VWF which even normal ADAMTS-13 level cannot cleave, and this may lead to TMA events. Future drugs targeting the VWF-platelet interaction could be efficient in transplant and malignancy-associated TMA.

\section{ASSAYS FOR ADAMTS-13 MEASUREMENT}

Several different types of assays are available for the measurement of ADAMTS-13: activity, antigen, inhibitor, and anti-ADAMTS-13 antibodies. The activity assays are most commonly reported in the literature; however, the measurement of antiADAMTS-13 antibodies is also highly important for the accurate TMA differentiation and TTP diagnosis (Table 1).

Functional assays measuring ADAMTS-13 activity are based on the ability of the patient plasma to degrade VWF multimers or synthetic VWF peptides. Inhibitory autoantibodies can be titrated in vitro using classical mixing studies, and neutralizing or non-neutralizing antibodies can be detected by Western blotting or enzyme-linked immunosorbent (ELISA) assays. ${ }^{65}$

Over the past few years, significant improvements have been made in ADAMTS-13 assays, and current commercially available tests allow more reproducible analyses with shorter turnaround time (1-4 hours), which can be conducted even in routine clinical diagnostic laboratories. ${ }^{66}$

\section{Activity Assays}

The original, or first-generation, assays for ADAMTS-13 activity measured the activity directly on the cleavage products, using VWF multimer analysis, or indirectly, using either collagen-binding or ristocetin aggregation assays. ${ }^{66}$ These methods were very time-consuming and available only in specialized and research laboratories. 
Table 1. ADAMTS-13 Levels and Pathophysiology in the Different Thrombotic Microangiopathies (TMAs).

\begin{tabular}{|c|c|c|c|}
\hline \multirow{2}{*}{ Type of TMA } & \multirow{2}{*}{ Pathophysiology } & \multicolumn{2}{|c|}{$\begin{array}{l}\text { ADAMTS-13 Level During } \\
\text { Acute Episode }\end{array}$} \\
\hline & & Activity & $\begin{array}{l}\text { Anti-ADAMTS-13 } \\
\text { Antibodies }\end{array}$ \\
\hline Congenital TTP & $\begin{array}{l}\text { ADAMTS- } 13 \text { deficiency causes UL-VWF- } \\
\text { platelet thrombi which lead to multi-organ } \\
\text { ischemic failure }\end{array}$ & $<5 \%$ & Absent \\
\hline Acquired TTP & $\begin{array}{l}\text { Antibodies against ADAMTS- } 13 \text { cause UL-VWF- } \\
\text { platelet thrombi which lead to multi-organ } \\
\text { ischemic failure }\end{array}$ & $<10 \%$ & Very High \\
\hline Shiga-HUS & $\begin{array}{l}\text { Shiga toxin causes secretion of UL-VWF and } \\
\text { the formation of VWF-platelet thrombi in the } \\
\text { glomerular microvasculature which lead to } \\
\text { acute renal failure }\end{array}$ & $>20 \%$ & Absent \\
\hline aHUS & $\begin{array}{l}\text { Dysregulation of the complement system, } \\
\text { mostly due to complement factor H } \\
\text { deficiency, leads to VWF-platelet thrombi in } \\
\text { the glomerular microvasculature and to } \\
\text { acute renal failure }\end{array}$ & $>20 \%$ & Absent \\
\hline $\begin{array}{l}\text { HELLP syndrome and pre- } \\
\text { eclampsia }\end{array}$ & $\begin{array}{l}\text { Abnormal and hypoxic placenta activates the } \\
\text { complement and coagulation cascades which } \\
\text { lead to thrombotic microangiopathy }\end{array}$ & $>20 \%$ & Absent \\
\hline $\begin{array}{l}\text { Transplant and malignancy- } \\
\text { associated TMAs }\end{array}$ & $\begin{array}{l}\text { Endothelial toxicity causes thrombotic } \\
\text { microangiopathy }\end{array}$ & $>20 \%$ & Absent \\
\hline DIC & $\begin{array}{l}\text { Disseminated intravascular coagulation } \\
\text { activation leads to thrombotic micro- } \\
\text { angiopathy and multi-organ ischemic failure }\end{array}$ & $>20 \%$ & Absent \\
\hline $\begin{array}{l}\text { Catastrophic } \\
\text { antiphospholipid syndrome }\end{array}$ & $\begin{array}{l}\text { Antiphospholipid antibodies cause } \\
\text { endothelial damage, thrombotic micro- } \\
\text { angiopathy, and multi-organ ischemic failure }\end{array}$ & $>20 \%$ & Absent \\
\hline
\end{tabular}

ADAMTS-13, a disintegrin and metalloproteinase with a thrombospondin type 1 motif, member 13; aHUS, atypical hemolytic uremic syndrome; DIC, disseminated intravascular coagulation; HELLP syndrome, hemolysis, elevated liver enzymes, and low platelets syndrome; Shiga-HUS, Shiga toxin-induced hemolytic uremic syndrome; TTP, thrombotic thrombocytopenic purpura; UL-VWF, ultra-large von Willebrand factor.

The first assay method was developed by Furlan et al. ${ }^{10}$ The substrate for this method was proteasefree plasma multimeric VWF. Detected plasma samples were first diluted, then activated by barium chloride, mixed with substrate, and dialyzed. Reaction products were separated by sodium dodecyl sulfate (SDS) agarose gel electrophoresis followed by immunoblotting. The resolution of the ladders of the degraded VWF multimers was high and reproducible, but the method required several days to complete. ${ }^{10,22}$

Newer techniques using both direct and indirect assays have been introduced that are more suitable for a routine testing laboratory. These assays utilize peptide substrates based on the ADAMTS-13 cleavage site in VWF, which contains the peptide bond between 1605Tyr and 1606Met in the A2 domain of VWF. These substrates are either a recombinant VWFA2 or synthesized VWF73 peptides. ${ }^{66}$

Patient plasma is incubated with the peptide substrate, and the residual VWF or cleavage product is measured by electrophoresis, fluorescence resonance energy transfer (FRET) technique, or immunoassay. Results are available within a few hours, and the improved methods allow higher throughput and have improved precision and sensitivity.

Multicenter studies found that, while the original assays based on multimeric VWF are sensitive (3\%- 
$6 \%$ of ADAMTS-13 activity) and reproducible, the newer assays based on VWF peptides are more sensitive ( $1 \%-3 \%$ of ADAMTS-13 activity), reproducible, easier, and rapid (1-4 h). ${ }^{66,67}$

According to the 2014-2 survey of the External Quality Control for Assays and Tests with a focus on Thrombosis and Haemostasis (ECAT Foundation), the majority of participants $(43 / 70)(61 \%)$ use ELISA method in ADAMTS-13 activity measurement with $13 \%$ coefficient of variation (CV); most of them (36/43) use the Technoclone Technozyme ADAMTS-13 activity kit. Only 24 (34\%) laboratories use the FRET method with $34 \%$ CV. ${ }^{68}$

\section{Anti-ADAMTS-13 Autoantibodies}

Two types of anti-ADAMTS-13 antibodies have been described: one inhibiting (neutralizing) ADAMTS-13 proteolytic activity ${ }^{17,18}$ and the other binding to the protease and accelerating its clearance from plasma through opsonization and/or other yet unresolved mechanisms. ${ }^{69}$ Both of these antibodies may be simultaneously present in many TTP patients. ${ }^{65}$

Neutralizing ADAMTS-13 autoantibodies (inhibitor) can be titrated in vitro using classic mixing studies of heat-inactivated patient and normal plasmas at a 1:1 dilution or several dilutions. However, although useful, Bethesda assays are far from being optimized and generally lack sensitivity. Less frequently (about 30\%), autoantibodies are non-neutralizing and probably promote the clearance of ADAMTS- 13 from blood without inhibiting its activity. ${ }^{69}$ These non-neutralizing antibodies can be detected using Western blotting or ELISA assays. 65

New assays use recombinant ADAMTS- 13 for the measurement of anti-ADAMTS-13 antibodies in a simplified ELISA. The detection wells are coated with recombinant ADAMTS-13. Antibodies against ADAMTS-13 from the detected plasma sample are recognized by conjugated anti-human IgG. The peroxidase level is determined by a chromogenic reaction that is proportional to the anti-ADAMTS-13 antibody level. The time for results with this method using the Technozyme ADAMTS-13 INH kit, (Technoclone, Vienna, Austria) is 2 hours 15 minutes.

Of 26 laboratories that participated in the 2014-2 survey of the ECAT Foundation, 19 (73\%) perform the anti-ADAMTS-13 antibodies measurement using the Technoclone Technozyme ADAMTS-13 INH kit, ${ }^{68}$ which is suitable for a rapid diagnosis in the routine clinical laboratory.

\section{Pre-analytical Variables of ADAMTS-13 Measurement Assays}

Blood samples for ADAMTS-13 activity and antibody should be drawn prior to treatment initiation, for the accurate assessment of baseline ADAMTS-13 levels. Knowledge of the timing of the treatment regime in terms of sample collection is important for the appropriate interpretation of ADAMTS-13 results. Plasma infusion of even one unit, which is often done in an attempt to stabilize patients with suspected TTP, may significantly change ADAMTS-13 levels. ${ }^{70}$

Samples should be collected into buffered sodium citrate anticoagulant tubes and should be centrifuged within 2 hours after collection for best results. Platelet-poor plasma should be tested after centrifuging at 3,000 $\mathrm{g}$ for 10 minutes or 2,000 $\mathrm{g}$ for 15 minutes. If plasma is not tested within 4 hours of collection, it should be re-centrifuged and separated off into a secondary aliquot tube for storage at below $-30^{\circ} \mathrm{C}$ for up to 3 months or below $-70^{\circ} \mathrm{C}$ for a longer period of time. Frozen plasma samples should be thawed rapidly at $37^{\circ} \mathrm{C}$ for 10 minutes in a water bath, mixed thoroughly before testing, and assayed within 4 hours. If not tested immediately after thawing, samples should be kept stored at 2$8^{\circ} \mathrm{C} .66$ Thawed samples should not be refrozen.

\section{The Need for ADAMTS-13 Measurement}

To make the accurate diagnosis of TTP and to differentiate it from other TMAs, blood samples must be drawn during the acute episode, prior to treatment initiation, since decreased levels of ADAMTS-13 activity (20\% < ADAMTS-13 < 50\%) can be detected also in other TMA events (Table 1). Severely reduced ADAMTS-13 activity $(<5 \%)$ during an acute episode, without presence of antiADAMTS-13 antibodies, supports the diagnosis of congenital TTP; whereas low ADAMTS-13 activity, in the presence of anti-ADAMTS-13 antibodies, confirms the diagnosis of acquired TTP. ${ }^{6}$

The specificity of severe ADAMTS-13 deficiency $(<5 \%)$ in distinguishing acute TTP from HUS is 90\%.36,71 This differentiation should be performed rapidly for the appropriate initiation of PEX for TTP and eculizumab for aHUS. 72

The diagnosis of acquired TTP with severely reduced ADAMTS-13 activity and elevated level of 
anti-ADAMTS-13 antibodies suggests a more intensive requirement for plasma therapy, increased mortality, and the risk of refractory disease which might need further immunosuppressive therapy.73

\section{Treatment Monitoring with ADAMTS-13}

While in congenital TTP it is probably sufficient to monitor plasma infusion efficiency by platelet and hemoglobin measurement only, in acquired TTP there is a benefit in monitoring the PEX efficacy by measuring the ADAMTS- 13 activity and antiADAMTS-13 antibody levels. $74-76$

Persistent presence of ADAMTS- 13 activity $<5 \%$ with elevated anti-ADAMTS- 13 antibodies during PEX therapy indicates the need for more frequent and prolonged PEX, and it may suggest the need to add immunosuppressive therapy. 77

Plasma exchange is effective in acute TTP through replacing deficient ADAMTS-13, removing associated antibody, and reducing circulating ULVWF multimers; however, in many patients, prolonged PEX is required to achieve remission, and between $30 \%$ and $60 \%$ of patients relapse over a variable period of months to years. 73 Therefore, the information about the ADAMTS-13 activity and antibodies can guide the decision regarding the appropriate therapy regimen for each patient. 77

\section{ADAMTS-13 as Predictor for Relapse and Survival}

Despite advances in TTP treatment, relapse occurs and mortality remains at $15 \%-20 \%$. Patients with persistent levels of ADAMTS- 13 activity of $<5 \%$ and the presence of elevated levels of anti-ADAMTS-13 antibody during an acute episode, and/or during remission, have a 3 -fold increased risk of relapse. ${ }^{46,78-80}$ Therefore, the identification of patients at the greatest risk for relapse can guide therapeutic decisions, such as the administration of prophylactic therapy or changing therapy to immunosuppressive agents to prevent relapse. 77

To date, monoclonal anti-CD20 therapy (rituximab) appears to be the most promising immunosuppressive treatment for patients with undetectable ADAMTS-13 activity or very high antiADAMTS-13 antibody levels with high risk for relapse. 77

It is noteworthy that while lower ADAMTS-13 activity in clinical remission predicts a risk of relapse, not all patients with severe ADAMTS-13 deficiency relapse. These data are consistent with previous reports suggesting that severely deficient ADAMTS-13 activity alone is not sufficient for the development of an acute episode of TTP, but rather an additional triggering factor or factors may be required. The search and identification of these triggering factors need to be continued to advance the TMAs management further.

\section{FUTURE PERSPECTIVES}

The accurate and urgent diagnosis of TMAs is exceedingly important because of their associated organ damage and mortality.

While progress has been made in the diagnosis and treatment of TTP, further improvements in therapeutic approaches and ADAMTS-13 assay standardization are required. Several potential therapies are in development for congenital and acquired TTP: 1) Recombinant ADAMTS-13.81,82 2) Gain-offunction ADAMTS-13 variants that resist inhibition by anti-ADAMTS-13 antibodies. ${ }^{83} 3$ ) Drugs targeting the interaction of A1 domain of VWF with platelet GPIb such as aptamers, anti-VWF nanobodies, or monoclonal antibody. ${ }^{84-86} 4$ ) Degradation or inhibition of UL-VWF. 87 These future promising therapies are in various stages of clinical trials.

In addition, international standards for the ADAMTS-13 assays are warranted to improve test sensitivity, specificity, and reliability, as well as inter-laboratory standardization and harmonization.

\section{ACKNOWLEDGEMENTS}

The author wishes to thank Professor B. Brenner, Professor J.M. Rowe, Dr N. Lanir, and Dr Y. Shechter for their support and encouragement to include the ADAMTS-13 evaluation in the hematology laboratory test repertoire; in addition, to thank all the hematologists of the Department of Hematology and Bone Marrow Transplantation at Rambam Health Care Campus and all the hematologists from all over Israel and especially to Professor E.J. Dann, who worked very closely with us and shared all the important clinical information. Thanks also go to Yevgenia Edelman, Kochava Maler, Etab Higazi, and Dr Anat Keren-Politansky for their skilled laboratory assistance; and special thanks, in gratitude, to Galila Tennenbaum, who has been walking with me all the way until we have become the first referral and central laboratory in Israel for ADAMTS-13 measurement. 
The author wishes also to acknowledge the External quality Control for Assays and Tests with a focus on Thrombosis and Haemostasis (ECAT) Foundation and Dr P. Meijer, the director of ECAT Foundation, for the permission to include the ADAMTS-13 results of the 2014-2 survey in this review. ${ }^{68}$ In addition, thanks go to the ECAT Foundation for conducting such an important external quality control scheme which measures the ADAMTS-13 proficiency tests among the different clinical diagnostic laboratories.

\section{REFERENCES}

1. Moake JL. Thrombotic microangiopathies. N Engl J Med 2002;347:589-600. Full Text

2. Tsai HM. The molecular biology of thrombotic microangiopathy. Kidney Int 2006;70:16-23. Full Text

3. Lämmle B, Kremer Hovinga JA, Alberio L. Thrombotic thrombocytopenic purpura. J Thromb Haemost 2005;3:1663-75. Full Text

4. Tsai HM. Autoimmune thrombotic microangiopathy: advances in pathogenesis, diagnosis, and management. Semin Thromb Hemost 2012;38:469-82. Full $\underline{\text { Text }}$

5. Moschcowitz E. Hyaline thrombosis of the terminal arterioles and capillaries: a hitherto undescribed disease. Proc N Y Pathol Soc 1924;24:1.

6. Gasser C, Gautier E, Steck A, Siebenmann RE, Oechslin R. Hemolytic-uremic syndrome: bilateral necrosis of the renal cortex in acute acquired hemolytic anemia. Schweiz Med Wochenschr 1955;85: 905-9.

7. Upshaw JD Jr. Congenital deficiency of a factor in normal plasma that reverses microangiopathic hemolysis and thrombocytopenia. N Engl J Med 1978;298: 1350-2. Full Text

8. Moake JL, Rudy CK, Troll JH, et al. Unusually large plasma factor VIII: von Willebrand factor multimers in chronic relapsing thrombotic thrombocytopenic purpura. N Engl J Med 1982;307:1432-5. Full Text

9. Karmali MA, Petric M, Lim C, Fleming PC, Arbus GS, Lior $\mathrm{H}$. The association between idiopathic hemolytic uremic syndrome and infection by verotoxinproducing Escherichia coli. J Infect Dis 1985;151: 775-82. Full Text

10. Furlan M, Robles R, Lamie B. Partial purification and characterization of a protease from human plasma cleaving von Willebrand factor to fragments produced by in vivo proteolysis. Blood 1996;87:4223-34.

11. Tsai HM. Physiologic cleavage of von Willebrand factor by a plasma protease is dependent on its con- formation and requires calcium ion. Blood 1996;87: 4235-44.

12. Fujikawa K, Suzuki H, McMullen B, Chung D. Purification of von Willebrand factor-cleaving protease and its identification as a new member of the metalloproteinase family. Blood 2001;98:1662-6. Full Text

13. Gerritsen HE, Robles R, Lammle B, Furlan M. Partial amino acid sequence of purified von Willebrand factor-cleaving protease. Blood 2001;98:1654-61. Full Text

14. Levy GG, Nichols WC, Lian EC, et al. Mutations in a member of the ADAMTS gene family cause thrombotic thrombocytopenic purpura. Nature 2001;413: 488-94. Full Text

15. Kokame K, Matsumoto M, Soejima K, et al. Mutations and common polymorphisms in ADAMTS13 gene responsible for von Willebrand factor-cleaving protease activity. Proc Natl Acad Sci U S A 2002;99: 11902-7. Full Text

16. Antoine G, Zimmermann $\mathrm{K}$, Plaimauer $\mathrm{B}$, et al. ADAMTS13 gene defects in two brothers with constitutional thrombotic thrombocytopenic purpura and normalization of von Willebrand factor-cleaving protease activity by recombinant human ADAMTS13. Br J Haematol 2003;120:821-4. Full Text

17. Furlan M, Robles R, Solenthaler M, Lammle B. Acquired deficiency of von Willebrand factor-cleaving protease in a patient with thrombotic thrombocytepenic purpura. Blood 1998;91:2839-46.

18. Tsai HM, Lian ECY. Antibodies to von Willebrand factor-cleaving protease in acute thrombotic thrombocytopenic purpura. N Engl J Med 1998;339:1585-94. Full Text

19. Turner N, Nolasco L, Nolasco J, Sartain S, Moake J. Thrombotic microangiopathies and the linkage between von Willebrand Factor and the alternative complement pathway. Semin Thromb Hemost 2014; 40:544-50. Full Text

20. Moake JL, Turner NA, Stathopoulos NA, et al. Involvement of large plasma von Willebrand factor (vWF) multimers and unusually large vWF forms derived from endothelial cells in shear stress-induced platelet aggregation. J Clin Invest 1986;78:1456-61. Full Text

21. Arya M, Anvari B, Romo GM, et al. Ultra-large multimers of von Willebrand factor form spontaneous high-strength bonds with the platelet GP Ib-IX complex: studies using optical tweezers. Blood 2002;99: 3971-7. Full Text

22. Sadler JE, Moake JL, Miyata T, George JN. Recent advances in thrombotic thrombocytopenic purpura. 
Hematology Am Soc Hematol Educ Program 2004: 407-23. Full Text

23. Moake J. Thrombotic thrombocytopenia purpura (TTP) and other thrombotic microangiopathies. Best Pract Res Clin Haematol 2009;22:567-76. Full Text

24. Tao Z, Peng Y, Nolasco L, et al. Recombinant CUB-1 domain polypeptide inhibits the cleavage of ULVWF strings by ADAMTS13 under flow conditions. Blood 2005;106:4139-45. Full Text

25. Dong JF, Moake JL, Bernardo A, et al. ADAMTS-13 metalloprotease interacts with the endothelial cellderived ultra-large von Willebrand factor. $\mathrm{J}$ Biol Chem 2003;278:29633-9. Full Text

26. Moake J. Thrombotic microangiopathies: multimers, metalloprotease, and beyond. Clin Transl Sci 2009;2: 366-73. Full Text

27. Scully M, Yarranton H, Liesner R, et al. Regional UK TTP registry: correlation with laboratory ADAMTS 13 analysis and clinical features. Br J Haematol 2008; 142:819-26. Full Text

28. Rock GA. Management of thrombotic thrombocytopenic purpura. Br J Haematol 2000;109:496-507 Full Text

29. Galbusera M, Noris M, Remuzzi G. Thrombotic thrombocytopenic purpura-then and now. Semin Thromb Hemost 2006;32:81-9. Full Text

30. Mansouri Taleghani M, von Krogh A-S, Fujimura Y, et al. Hereditary thrombotic thrombocytopenic purpura and the hereditary TTP registry. Hämostaseologie 2013;2:138-43. Full Text

31. Camilleri RS, Scully M, Thomas M, et al. A phenotype-genotype correlation of ADAMTS13 mutations in congenital thrombotic thrombocytopenic purpura patients treated in the United Kingdom. J Thromb Haemost 2012;10:1792-801. Full Text

32. Scully M, Gattens M, Khair K, Liesner R. The use of intermediate purity factor VIII concentrate BPL $8 \mathrm{Y}$ as prophylaxis and treatment in congenital thrombotic thrombocytopenic purpura. Br J Haematol 2006; 135:101-4. Full Text

33. Schiff DE, Roberts WD, Willert J, Tsai HM. Thrombocytopenia and severe hyperbilirubinemia in the neonatal period secondary to congenital thrombotic thrombocytopenic purpura and ADAMTS13 deficiency. J Pediatr Hematol Oncol 2004;26:535-8. Full Text

34. Loirat C, Veyradier A, Girma JP, Ribba AS, Meyer D. Thrombotic thrombocytopenic purpura associated with von Willebrand factor cleaving protease (ADAMTS13) deficiency in children. Semin Thromb Hemost 2006;32:90-7. Full Text
35. Rosove MH. Thrombotic microangiopathies. Semin Arthritis Rheum 2014;43:797-805. Full Text

36. Scully M, Hunt BJ, Benjamin S, et al. British Committee for Standards in Haematology. Guidelines on the diagnosis and management of thrombotic thrombocytopenic purpura and other thrombotic microangiopathies. Br J Haematol 2012;158:323-35. Full Text

37. Lotta LA, Wu HM, Mackie IJ, et al. Residual plasmatic activity of ADAMTS 13 is correlated with phenotype severity in congenital thrombotic thrombocytopenic purpura. Blood 2012;120:440-8. Full Text

38. Moatti-Cohen M, Garrec C, Wolf M, et al. Unexpected frequency of Upshaw-Schulman syndrome in pregnancy-onset thrombotic thrombocytopenic purpura. Blood 2012;119:588-97. Full Text

39. Furlan M, Robles R, Solenthaler M, Wassmer M, Sandoz P, Lammle B. Deficient activity of von Willebrand factor-cleaving protease in chronic relapsing thrombotic thrombocytopenic purpura. Blood 1997; 89:3097-103.

40. Furlan M, Robles R, Galbusera M, et al. von Willebrand factor-cleaving protease in thrombotic thrombocytopenic purpura and the hemolytic-uremic syndrome. N Engl J Med 1998;339:1578-84. Full Text

41. Schneppenheim R, Budde U, Oyen F, et al. von Willebrand factor cleaving protease and ADAMTS13 mutations in childhood TTP. Blood 2003;101:184550. Full Text

42. Fujimura Y, Matsumoto M, Isonishi A, et al. Natural history of Upshaw-Schulman syndrome based on ADAMTS13 gene analysis in Japan. J Thromb Haemost 2011;9(Suppl 1):283-301. Full Text

43. Terrell DR, Williams LA, Vesely SK, Lämmle B, Hovinga JA, George JN. The incidence of thrombotic thrombocytopenic purpura-hemolytic uremic syndrome: all patients, idiopathic patients, and patients with severe ADAMTS-13 deficiency. $J$ Thromb Haemost 2005;3:1432-6. Full Text

44. Douglas KW, Pollock KG, Young D, Catlow J, Green R. Infection frequently triggers thrombotic microangiopathy in patients with preexisting risk factors: a single-institution experience. J Clin Apher 2010;25: 47-53.

45. George JN. The association of pregnancy with thrombotic thrombocytopenic purpura-hemolytic uremic syndrome. Curr Opin Hematol 2003;10:33944. Full Text

46. Kremer Hovinga JA, Vesely SK, Terrell DR, Lämmle B, George JN. Survival and relapse in patients with thrombotic thrombocytopenic purpura. Blood 2010; 115:1500-11. Full Text 
47. Bell WR, Braine HG, Ness PM, Kickler TS. Improved survival in thrombotic thrombocytopenia purpurahemolytic uremic syndrome-clinical experience in 108 patients. N Engl J Med 1991;325:398-403. Full $\underline{\text { Text }}$

48. Kennedy AS, Lewis QF, Scott JG, et al. Cognitive deficits after recovery from thrombotic thrombocytopenic purpura. Transfusion 2009;49:1092-101. Full Text

49. Deford CC, Reese JA, Schwartz LH, et al. Multiple major morbidities and increased mortality during long-term follow-up after recovery from thrombotic thrombocytopenic purpura. Blood 2013;122:2023-9. Full Text

50. George JN. How I treat patients with thrombotic thrombocytopenic purpurahemolytic uremic syndrome. Blood 2010;116:4060-9. Full Text

51. Mele C, Remuzzi G, Noris M. Hemolytic uremic syndrome. Semin Immunopathol 2014;36:399-420. Full Text

52. Nolasco LH, Turner NA, Bernardo A, et al. Hemolytic uremic syndrome-associated Shiga toxins promote endothelial-cell secretion and impair ADAMTS13 cleavage of unusually large von Willebrand factor multimers. Blood 2005;106:4199-209. Full Text

53. Frank C, Werber D, Cramer JP, et al. Epidemic profile of Shiga-toxin-producing Escherichia coli O104:H4 outbreak in Germany. N Engl J Med 2011;365:1771-80. Full Text

54. Noris M, Remuzzi G. Atypical hemolytic-uremic syndrome. N Engl J Med 2009; 361:1676-87. Full Text

55. Mutter WP, Karumanchi SA. Molecular mechanisms of preeclampsia. Microvasc Res 2008;75:1-8. Full Text

56. Ducloy-Bouthors AS, Caron C, Subtil D, et al. Thrombotic thrombocytopenic purpura: medical and biological monitoring of six pregnancies. Eur J Obstet Gynecol Reprod Biol 2003;111:146-52. Full Text

57. Scully M, Starke R, Lee R, Mackie I, Machin S, Cohen $\mathrm{H}$. Successful management of pregnancy in women with a history of thrombotic thrombocytopaenic purpura. Blood Coagul Fibrinolysis 2006;17:459-63. Full $\underline{\text { Text }}$

58. Jacob S, Dunn BL, Qureshi ZP, et al. Ticlopidine-, clopidogrel-, and prasugrel-associated thrombotic thrombocytopenic purpura: a 20-year review from the Southern Network on Adverse Reactions (SONAR). Semin Thromb Hemost 2012;38:845-53. Full Text

59. Bennett CL, Kim B, Zakarija A, et al. Two mechanistic pathways for thienopyridine-associated thrombotic thrombocytopenic purpura: a report from the SERF-
TTP Research Group and the RADAR Project. J Am Coll Cardiol 2007;50:1138-43. Full Text

6o. Tsai HM, Rice L, Sarode R, Chow TW, Moake JL. Antibody inhibitors to von Willebrand factor metalloproteinase and increased binding of von Willebrand factor to platelets in ticlopidine-associated thrombotic thrombocytopenic purpura. Ann Intern Med 2000;132:794-9. Full Text

61. Sugio Y, Okamura T, Shimoda K, et al. Ticlopidineassociated thrombotic thrombocytopenic purpura with an IgG-type inhibitor to von Willebrand factorcleaving protease activity. Int J Hematol 2001;74: 347-51. Full Text

62. Ruutu T, Barosi G, Benjamin RJ, et al. Diagnostic criteria for hematopoietic stem cell transplant-associated microangiopathy: results of a consensus process by an International Working Group. Haematologica 2007;92:95-100. Full Text

63. Kwaan HC, Gordon LI. Thrombotic microangiopathy in the cancer patient. Acta Haematol 2001;106:52-6. Full Text

64. Fontana S, Gerritsen HE, Kremer HJ, Furlan M, Lammle B. Microangiopathic haemolytic anaemia in metastasizing malignant tumours is not associated with a severe deficiency of the von Willebrand factorcleaving protease. Br J Haematol 2001;113:100-2. Full Text

65. Peyvandi F, Palla R, Lotta LA, Mackie I, Scully MA, Machin SJ. ADAMTS-13 assays in thrombotic thrombocytopenic purpura. J Thromb Haemost 2010;8: 631-40. Full Text

66. Just S. Methodologies and clinical utility of ADAMTS-13 activity testing. Semin Thromb Hemost 2010;36:82-90. Full Text

67. Tripodi A, Peyvandi F, Chantarangkul V, et al. Second international collaborative study evaluating performance characteristics of methods measuring the von Willebrand factor cleaving protease (ADAMTS-13). J Thromb Haemost 2008;6:1534-41. Full Text

68. External Quality Control for Assays and Tests with a Focus on Thrombosis and Haemostasis. 2014-2 survey report. ECAT Foundation. July 30, 2014. Accessible to registered users via http://www.ecat.nl/

69. Scheiflinger F, Knobl P, Trattner B, et al. Nonneutralizing $\operatorname{IgM}$ and $\operatorname{IgG}$ antibodies to von Willebrand factor-cleaving protease (ADAMTS-13) in a patient with thrombotic thrombocytopenic purpura. Blood 2003;102:3241-3. Full Text

70. Knovich MA, Craver K, Matulis MD, Lawson H, Owen J. Simplified assay for VWF cleaving protease (ADAMTS13) activity and inhibitor in plasma. Am J Hematol 2004;76:286-90. Full Text 
71. Bianchi V, Robles R, Alberio L, Furlan M, Lämmle B. Von Willebrand factor-cleaving protease (ADAMTS13) in thrombocytopenic disorders: a severely deficient activity is specific for thrombotic thrombocytopenic purpura. Blood 2002;100:710-13. Full Text

72. Zuber J, Fakhouri F, Roumenina LT, Loirat C, Frémeaux-Bacchi V. French Study Group for aHUS/ $\mathrm{C}_{3} \mathrm{G}$. Use of eculizumab for atypical haemolytic uraemic syndrome and $\mathrm{C}_{3}$ glomerulopathies. Nat Rev Nephrol 2012;8:643-57. Full Text

73. Scully M. Inhibitory anti-ADAMTS 13 antibodies: measurement and clinical application. Blood Rev 2010;24:11-16. Full Text

74. Kappers-Klunne MC, van Asten JG, van Vliet HH. ADAMTS-13 and Von Willebrand factor in relation to platelet response during plasma exchange in thrombotic thrombocytopenic purpura: a clue for disease mechanism? Ann Hematol 2009;88:1025-8. Full Text

75. Starke R, Machin S, Scully M, Purdy G, Mackie I. The clinical utility of ADAMTS13 activity, antigen and autoantibody assays in thrombotic thrombocytopenic purpura. Br J Haematol 2006;136:649-55. Full Text

76. Loirat C, Coppo P, Veyradier A. Thrombotic thrombocytopenic purpura in children. Curr Opin Pediatr 2013;25:216-24. Full Text

77. Scully M. Rituximab in the treatment of TTP. Hematology 2012;17(Suppl 1):S22-4.

78. Peyvandi F, Lavoretano S, Palla R, et al. ADAMTS13 and anti-ADAMTS13 antibodies as markers for recurrence of acquired thrombotic thrombocytopenic purpura during remission. Haematologica 2008;93: 232-9. Full Text

79. Cataland SR, Wu HM. Practical issues in ADAMTS13 testing and emerging therapies in thrombotic thrombocytopenic purpura. Semin Hematol 2011; 48:242-50. Full Text 8o. Bettoni G, Palla R, Valsecchi C, et al. ADAMTS-13 activity and autoantibodies classes and subclasses as prognostic predictors in acquired thrombotic thrombocytopenic purpura. J Thromb Haemost 2012;10: 1556-65. Full Text

81. Schiviz A, Wuersch K, Piskernik C, et al. A new mouse model mimicking thrombotic thrombocytopenic purpura: correction of symptoms by recombinant human ADAMTS13. Blood 2012;119:6128-35. Full $\underline{\text { Text }}$

82. Plaimauer B, Kremer Hovinga JA, Juno C, et al. Recombinant ADAMTS13 normalizes von Willebrand factor-cleaving activity in plasma of acquired TTP patients by overriding inhibitory antibodies. J Thromb Haemost 2011;9:936-44. Full Text

83. Jian C, Xiao J, Gong L, et al. Gain-of-function ADAMTS13 variants that are resistant to autoantibodies against ADAMTS13 in patients with acquired thrombotic thrombocytopenic purpura. Blood 2012;119:3836-43. Full Text

84. Cataland SR, Peyvandi F, Mannucci PM, et al. Initial experience from a double blind, placebo-controlled, clinical outcome study of ARC1779 in patients with thrombotic thrombocytopenic purpura. Am J Hematol 2012;87:430-2. Full Text

85. Callewaert F, Roodt J, Ulrichts H, et al. Evaluation of efficacy and safety of the anti-VWF nanobody ALX0681 in a preclinical baboon model of acquired thrombotic thrombocytopenic purpura. Blood 2012; 120:3603-10. Full Text

86. Feys HB, Roodt J, Vandeputte N, et al. Inhibition of von Willebrand factor platelet glycoprotein Ib interaction prevents and reverses symptoms of acute acquired thrombotic thrombocytopenic purpura in baboons. Blood 2012;120:3611-14. Full Text

87. Chen J, Reheman A, Gushiken FC, et al. Nacetylcysteine reduces the size and activity of von Willebrand factor in human plasma and mice. J Clin Invest 2011;12:593-603. Full Text 